COVID-19 AND THE KIDNEYS IN 2021

\section{The COVID-19 pandemic: progress in nephrology}

\section{María José Soler (1) and Conxita Jacobs-Cachá}

Patients with kidney disease are particularly vulnerable to COVID-19. In 2021, key studies demonstrated the safety of renin-angiotensin blockade in patients with kidney failure and COVID-19, and provided new data on the therapeutic potential of soluble angiotensin-converting enzyme, COVID-19 vaccine responses and the long-term effects of COVID-19 on kidney function.

In the past year, the COVID-19 pandemic has continued to have an important impact on everyday life and on the management of kidney disease. Early in the pandemic, the finding that SARS-CoV-2 uses angiotensin-converting enzyme 2 (ACE2) as a receptor to enter target cells led to concerns that use of ACE inhibitors or angiotensin receptor blockers (ARBs) might increase the risk of severe COVID-19 ${ }^{1}$. Several observational studies and randomized clinical trials reported no deleterious effect of renin-angiotensin system (RAS) blockade on the risk of SARS-CoV-2 infection or COVID-19 severity in patients with hypertension ${ }^{2}$. However, the effects in patients with kidney failure, who often use RAS blockers, were unknown. In late 2021, an analysis of data from the European Renal Association COVID-19 Database (ERACODA) demonstrated no significant association between previous use or continuation of RAS blockade and 28-day mortality among patients on dialysis and kidney transplant recipients with COVID-19 after adjustment for baseline demographics, comorbidities and COVID-19 severity ${ }^{3}$. In addition, RAS blockade was not associated with a substantially increased risk of hospital admission, intensive care unit admission or need for ventilator support in these patients. This study indicates that RAS blockade can be safely continued in patients with kidney failure and COVID-19.

The role of ACE2 in SARS-CoV-2 infection has also led to investigation of this receptor as a potential therapeutic target. As SARS-CoV-2 binds to membrane-bound full-length ACE2, Wysocki et al. investigated whether a truncated form of human soluble ACE2 could be used as a decoy receptor to limit viral infection ${ }^{4}$. The truncated protein (ACE2 1-618) was fused with an albumin-binding domain (ABD) to increase its duration of action. In mice, plasma ACE2 1-618-ABD had substantial catalytic activity $96 \mathrm{~h}$ after injection and remained biologically active (that is, retained its ability to lower blood pressure when given 3 days before angiotensin II injection). Wysocki et al. also showed that human kidney organoids express both full-length ACE2 and TMPRSS2 protease, which is required for fusion and internalization of the ACE2-SARS-CoV-2 viral spike protein complex. ACE2 1-618-ABD neutralized SARS-CoV-2 infection in these organoids. In a preprint, the researchers demonstrate that treatment with a dimeric version of the ACE2 1-618-ABD construct (ACE2 1-618$\mathrm{DDD}-\mathrm{ABD}$ ) protected a transgenic mouse model of lethal SARS-CoV-2 infection from developing severe disease ${ }^{5}$. A case report ${ }^{6}$ of successful treatment of a patient with severe COVID-19 using full-length soluble recombinant human ACE2 (rhACE2) was published in 2020, and this therapy is now being studied in a clinical trial ${ }^{7}$. Wysocki et al. suggest that their truncated ACE2 construct might be more suitable than rhACE2 for treatment of COVID-19 because of its longer duration of action and smaller size, which should enable it to be filtered by the kidney and potentially protect against COVID-19-associated kidney disease $^{4}$.

Vaccination is one of the most important weapons in the fight against SARS-CoV-2. Patients on dialysis and kidney transplant recipients often have impaired immune responses and therefore require higher and more frequent vaccine doses than the general population. In 2021, Bensouna et al. studied the humoral response to a third dose of the BNT162b2 mRNA COVID-19 vaccine among patients on haemodialysis or peritoneal dialysis $(n=69)^{8}$. They report that administration of a third vaccine dose increased the levels of anti-SARS-CoV-2 spike protein S1 (anti-S1) antibodies from a median of 284
Vaccination is one of the most important weapons in the fight against SARS-CoV-2

(interquartile range, 83-1,190) arbitrary units $/ \mathrm{mL}$ at a median of 50 days after the second dose to a median of 7,554 (interquartile range, 2,268-11,736) arbitrary units/mL at a median of 30 days after the third dose. The vaccine reactions were acceptable and overall self-reported tolerance to the third dose was similar to that of the second dose. The greatest increases in antibody levels following a third dose of the BNT162b2 vaccine were seen in patients who had lower antibody levels after the second dose and longer intervals between the second and third doses. By contrast, little evidence of an increase in antibody level after the third dose was seen in patients who had high anti-S1 antibody levels after the second dose or in those who were undergoing chemotherapy. Other factors that were associated with a poor antibody response after three doses of vaccine included a history of immunosuppressive therapy or haemopathy, lower serum $\gamma$-globulin and initial lower anti-S1 antibodies after two doses. These results suggest that booster SARS-CoV-2 vaccine administration in patients on dialysis should be individualized depending on the initial anti-S1 antibody levels after the second vaccine dose.

Following the acute phase of COVID-19, some patients develop post-COVID-19 syndrome (also known as long COVID). Symptoms of this disorder include fatigue, dyspnoea, chest pain, cognitive disturbances, arthralgia and decline in quality of life. In 2021, one of the largest studies yet of the long-term health consequences of

Key advances
- Renin-angiotensin system blockade is not
associated with increased risk or severity
of COVID-19 among patients with kidney
failure ${ }^{3}$.
- A truncated form of human soluble
angiotensin-converting enzyme 2
(ACE2) protected kidney organoids from
SARS-CoV-2 infection ${ }^{4}$ this protein might
be a potential preventive or therapeutic
agent for COVID-19.
In patients with kidney failure, a third dose
of the BNT162b2 COVID-19 vaccine was
well tolerated and substantially increased
anti-SARS-CoV-2 antibodies ${ }^{8}$
A proportion of patients (13-35\%) experience
a decline in estimated glomerular filtration
rate in the long term following severe
COVID-199; thus, COVID-19 should be an
indication for kidney disease screening.


a Safety of RAS blockade

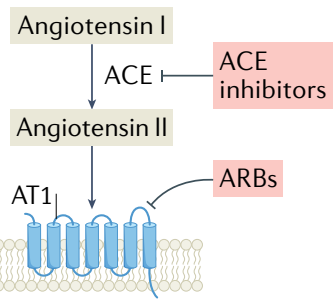

b Potential novel therapy SARS-CoV-2

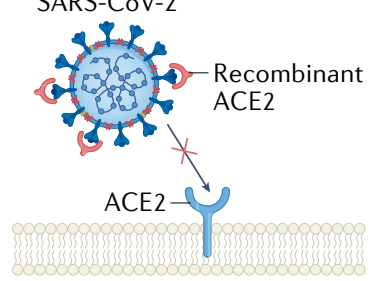

c Vaccination strategy

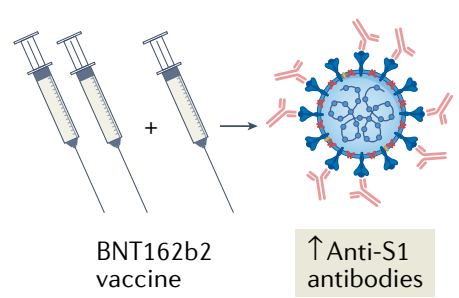

d Long-term effects on the kidney

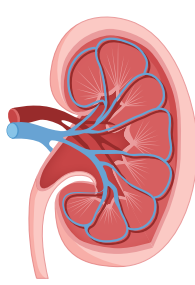

- 6\% of patients
develop AKI during
acute COVID-19
infection
- $13-35 \%$ of patients
show decline in
eGFR 6 months
after onset of
COVID-19
symptoms

Fig. 1 | New knowledge of COVID-19 and the kidneys in 2021. a | Renin-angiotensin system (RAS) blockade with either angiotensinconverting enzyme (ACE) inhibitors or angiotensin receptor blockers (ARBs) is not associated with increased risk or severity of COVID-19 in patients with kidney failure. $\mathbf{b} \mid$ Recombinant ACE2 protein could be a potential therapeutic agent to prevent or treat COVID-19. $c \mid A$ third dose of the BNT162b2 COVID-19 vaccine increases anti-SARS-CoV-2 spike protein S1 (anti-S1) antibodies in patients with kidney failure on dialysis. d | In addition to the development of acute kidney injury (AKI) during acute COVID-19, the disease can lead to loss of kidney function in the long term. AT1, angiotensin II receptor type 1; eGFR, estimated glomerular filtration rate.
COVID-19 reported loss of kidney function among patients with COVID-19 who had been discharged from a hospital in Wuhan, China'. In this study, 101 of 1,706 patients (6\%) developed acute kidney injury during the acute phase of COVID-19. The incidence of decreased estimated glomerular filtration rate $\left(\mathrm{eGFR}<90 \mathrm{ml} / \mathrm{min} / 1.73 \mathrm{~m}^{2}\right)$ at 6 months after onset of COVID-19 symptoms varied from $13 \%$ among patients who did not develop acute kidney injury and had normal eGFR during the acute phase to $35 \%$ among all participants who had eGFR data available during the acute phase and follow-up period. These findings suggest that severe COVID-19 requiring hospitalization should be an indication for kidney disease screening.

Severe COVID-19 requiring hospitalization should be an indication for kidney disease screening
In the past year, these key studies have contributed to a rapid increase in knowledge regarding COVID-19 and the kidney (FIG. 1). The new data will help to develop strategies to prevent and manage COVID-19 among patients with kidney disease, as well as to reduce the long-term adverse effects of COVID-19 on the kidney.

María José Soler (iD $\bowtie$ and Conxita Jacobs-Cachá Nephrology Department, Vall d'Hebron University Hospital, Vall d'Hebron Research Institute, Barcelona, Spain

凶e-mail:mjsoler01@gmail.com

https://doi.org/10.1038/s41581-021-00521-4

1. Sparks, M. A. et al. Sound science before quick judgement regarding RAS blockade in COVID-19. Clin. J. Am. Soc. Nephrol. 15, 714-716 (2020).

2. Soler, M. J. et al. Association of renin-angiotensin system blockers with COVID-19 diagnosis and prognosis in patients with hypertension: a populationbased study. Clin. Kidney J. https://doi.org/10.1093/ ckj/sfab 161 (2021).

3. Soler, M. J. et al. Renin-angiotensin system blockers and the risk of COVID-19-related mortality in patients with kidney failure. Clin. J. Am. Soc. Nephrol. 16, 1061-1072 (2021).

4. Wysocki, J. et al. A novel soluble ACE2 variant with prolonged duration of action neutralizes SARS-CoV-2 infection in human kidney organoids. J. Am. Soc. Nephrol. 32, 795-803 (2021).

5. Hassler, L. et al. A novel soluble ACE2 protein totally protects from lethal disease caused by SARS-CoV-2 infection. Preprint at bioRxiv https://doi.org/10.1101/ 2021.03.12.435191 (2021).

6. Zoufaly, A. et al. Human recombinant soluble ACE2 in severe COVID-19. Lancet Respir. Med. 8, 1154-1158 (2020).

7. US National Library of Medicine. ClinicalTrials.gov https://clinicaltrials.gov/ct2/show/NCT0 4335136 (2021).

8. Bensouna, I. et al. SARS-CoV-2 antibody response after a third dose of the BNT162b2 vaccine in patients receiving maintenance hemodialysis or peritoneal dialysis. Am. J. Kidney Dis. https://doi.org/10.1053/J.AJKD.2021.08.005 (2021).

9. Huang, C. et al. 6-month consequences of COVID-19 in patients discharged from hospital: a cohort study. Lancet 397, 220-232 (2021).

\section{Acknowledgements}

The authors' work was supported by a grant from the Fondo de Investigación Sanitaria-Feder, ISCIII, PI17/00257, RICORS, RD21/0005/0016, Marató TV3 2021, 215/C/2021 and EIN2020-112338.

\section{Competing interests}

M.J.S. reports grants and personal fees from Novo Nordisk, Janssen, Boehringer, Eli Lilly, AstraZeneca, Esteve, FMC, Mundipharma, Vifor, Bayer, Travere Therapeutics, GE Healthcare and $\mathrm{UCl}$ outside the submitted work. C.J.C. reports a grant from Travere Therapeutics outside the submitted work. 